\title{
A Subtracted cDNA Library from the Zebrafish (Danio rerio) Embryonic Inner Ear
}

\author{
Roney S. Coimbra, ${ }^{1}$ Dominique Weil, ${ }^{1}$ Phillipe Brottier, ${ }^{2}$ Stéphane Blanchard, ${ }^{1}$ \\ Michael Levi, ${ }^{2}$ Jean-Pierre Hardelin, ${ }^{1}$ Jean Weissenbach, ${ }^{2}$ and Christine Petit ${ }^{1,3}$ \\ ${ }^{1}$ Unité de Génétique des Déficits Sensoriels, Centre National de la Recherche Scientifique Unité de Recherche Associér (URA) \\ 1968, Institut Pasteur, 75724 Paris cedex 15, France; ${ }^{2}$ Génoscope, Centre National de Séquençage, 91006 Évry cedex, \\ France
}

\begin{abstract}
A database was built that consists of 4694 sequence contigs of $\sim 18,000$ reads of cDNAs isolated from the microdissected otocysts of zebrafish embryos at 20-30 hour postfertilization, following subtraction with a pool of liver cDNAs from adult fish. These sequences were compared with those of public databanks. Significant similarity were recorded and organized in a relational database at http://www.genoscope.cns.fr/zie. A first group of 2067 sequences correspond to 1428 known zebrafish genes or ESTs present in the Danio rerio section of UniGene. A second group of 302 sequences encode putative proteins that showed significant similarity (50\%-100\%) with 302 nonzebrafish proteins in the $\mathrm{nr}$ databank, a public databank containing an exhaustive nonredundant collection of protein sequences from different species (ftp://ftp.ncbi.nlm.nih.gov/blast $/ \mathrm{db} / \mathrm{hr}$ ). The remaining 2325 (49.5\%) sequence contigs or singletons showed no significant similarity with sequences available in public databanks. Several genes known to be expressed in the developing inner ear were represented in the present database, in particular genes involved in hair cell differentiation or innervation The occurrence of these genes validates the outcome of this study as the first collection of ESTs preferentially expressed in the zebrafish inner ear during the period of hair cell differentiation and neuroblast delamination from the otic vesicle epithelium. Novel zebrafish genes also involved in these processes are thus likely to be represented among the sequences obtained herein, for which no homology was found in the D. rerio section of UniGene.
\end{abstract}

[The sequence data from this study have been submitted to EMBL under accession nos. AL714032-AL731531].

The establishment of the structure of the inner ear is under the influence of genes controlling complex networks of molecular interactions. Although several genes implicated in inner ear development have been identified in recent years (for review, see Torres and Giráldez 1998), the puzzle is still far from being assembled. Mouse mutants with behavioral abnormalities have been a great aid in the identification and isolation of a large number of genes expressed in the developing ear (Deol 1968, 1970, 1980; Steel 1995). However, in recent years, zebrafish (Danio rerio), an aquarium fish originating from the rivers of tropical India, has become a favorite animal model due to a rare combination of attractive features, including large progenies, external fertilization and embryonic development, embryo transparency, can obtain haploid and homozygous diploid individuals by gynogenesis (Streisinger et al. 1981, 1986; Streisinger 1984), and so on.

A zebrafish genome-sequencing project has just begun at the Sanger Centre. Genetic linkage maps now cover the entire zebrafish genome (Knapik et al. 1998; Postlethwait et al. 1998; Gates et al. 1999; Shimoda et al. 1999). In addition, two independent physical maps made from zebrafish-rodent radiation hybrid lines (LN54 and T51) are available, which to-

\section{${ }^{3}$ Corresponding author.}

E-MAIL cpetit@pasteur.fr; FAX 33-1-45-67-69-78.

Article and publication are at http://www.genome.org/cgi/doi/10.1101/ gr.227502. gether cover $>90 \%$ of the zebrafish genome (Geisler et al. 1999; Hukriede et al. 1999).

Concomitantly, large-scale screenings for embryogenesis defects in zebrafish mutants have been carried out, which have permitted the identification of mutants with defects of the ear development. Twenty of these mutants, defining 13 independent loci, have been phenotypically and genetically characterized by Malicki et al. (1996). In an independent study, 95 mutants (39 genes) showing defects in inner ear development have been reported by Whitfield et al. (1996) among zebrafish mutants previously identified through a large-scale mutagenesis screening (Haffter et al. 1996). Another large-scale screening in zebrafish was announced by Amsterdam et al. (1999). The strategy of insertional mutagenesis proposed by this group is expected to accelerate the isolation of the disrupted genes.

Despite some differences in the ear structure of otophysan compared to mammals (otophysan species, including zebrafish, have no middle or outer ear structure and they possess two sound-sensitive maculae in the inner ear instead of the mammalian organ of Corti), the hair cells, the sensory cells that detect sound waves and acceleration are remarkably conserved (Platt 1993; Popper and Fay 1993). The screening of zebrafish mutants for balance phenotypes combined with a candidate gene cloning approach is a potentially powerful strategy for revealing some of the genes that play a crucial role in the developing and functioning of hair cells. However, so 
far, only the myosin VIIA gene, which is defective in mariner zebrafish mutants, has been isolated (Ernest et al. 2000) on the basis of the involvement of its orthologs in balance defects and/or deafness in mice (Gibson et al. 1995) and humans (Weil et al. 1995). Improvement of the candidate gene approach requires a more extensive characterization of the genes expressed in the inner ear.

This study is the first large-scale sequencing project aimed at gaining information on gene expression in the inner ear of developing zebrafish. The inner ear of zebrafish and other vertebrates differentiates from the otic placode, a dorsolateral thickening of the surface ectoderm, adjacent to the rhombencephalon (Waterman and Bell 1984; Platt 1993; Haddon and Lewis 1996). In the zebrafish, the otic placode can be identified at $16 \mathrm{hr}$ postfertilization (hpf). The placode gives rise to the otic vesicle (at $18 \mathrm{hpf}$ ), from which neuroblasts, the precursors of sensory neurons, will later delaminate to form the vestibuloacoustic ganglion. The peak of neuroblast delamination is between 22 and $30 \mathrm{hpf}$. At $\sim 24 \mathrm{hpf}$, the first hair cells differentiate within two sensory maculae. The three semicircular canals form between 43 and $72 \mathrm{hpf}$ from the walls of the otocyst, and three additional clusters of hair cells soon become visible in the sensory cristae of these canals. Thus, by the end of the first week, all key structures of the ear are present, but before the ear is completely mature, thousands more hair cells and neurons will be produced by subsequent division of precursors (Waterman and Bell 1984; Haddon and Lewis 1996). Specifically, we focused this study on the 20-30 hpf stage, corresponding to the period of differentiation of the first hair cells and to the peak of neuroblast delamination (Waterman and Bell 1984; Haddon and Lewis 1996). The strategy of cDNA library subtraction was chosen because it has already been successful in identifying genes preferentially or specifically expressed in the human, mouse, and chicken auditory sensory organ (Robertson et al. 1994, 1998; Cohen-Salmon et al. 1997; Heller et al. 1998; Yasunaga et al. 1999; Simmler et al. 2000; Verpy et al. 2000, 2001). A pool of cDNAs from the liver was used as driver to minimize representation of housekeeping genes in the subtracted library.

\section{RESULTS AND DISCUSSION}

After trimming and assembling (see Methods) the 17,468 individual reads of $>100$-bp long, the final database contained 4694 contigs and singletons averaging $530 \mathrm{bp}$. Forty-four percent $(2067 / 4694)$ of these sequences matched at least one $D$. rerio sequence cluster in the UniGene databank (Table 1 ).

A total of $1428 D$. rerio UniGene clusters were significantly similar to 2067 sequences in our database. Sixty-one percent $(880 / 1428)$ corresponded to $D$. rerio known genes or to $D$. rerio ESTs previously classified as highly, moderately, or weakly similar to known (or predicted) genes in other organisms. A number of these genes are known to be expressed in the developing inner ear of zebrafish or other vertebrates during the early stage of hair cell differentiation (Holme et al. 2002). These include the genes encoding the transcription factors msxc, pax2, eya1, dlx2, and genes encoding signaling molecules that drive hair cell fate determination and proliferation (deltaA, deltaB, and deltaD, Lunatic fringe, and retinoic acid receptor gamma). The gene encoding the transcription factor pax 2 is expressed early in the otic placode, and its expression is maintained in a restricted region of the otic vesicle. Whereas eya1, encoding a transcription coactivator
Table 1. Analysis of Clone Sequences

n (\%)

Total reads generated from independent clones 21848 Reads selected for further study 17468

Total of contigs and singletons after clustering

Sequences matching previously described Dario rerio sequences

Sequences matching proteins in nonredundant

$4694(100 \%)$

equences with no match

$2067(44 \%)$ $302(6.5 \%)$ $2325(49.5 \%)$

underlying the branchio-oto-renal syndrome (Abdelhak et al. 1997; Kalatzis et al. 1998), and pax2 are both expressed in the ventro-medial wall of the otic vesicle (i.e., the presumptive area of the sensory epithelia; Rinkwitz-Brandt et al. 1996; Sahly et al. 1999), the homeobox-containing gene msxc is expressed in the dorso-lateral aspect of the vesicle. Msxc continues to be expressed during the differentiation period, and eya1 persists even after differentiation has taken place (Ekker et al. 1992; Sahly et al. 1999). Finally, $d l x 2$ is expressed in the sensory patches during the period of hair cell differentiation (Robinson and Mahon 1994). Several different families of signaling molecules have been shown to be involved in the generation and maintenance of the complex cell architecture patterns of hair cells and supporting cells in the sensory epithelia. The notch-signaling pathway regulates sensory cell commitment in the vertebrate inner ear. In zebrafish, the delta homologs delta $A$, deltaB, and deltaD, coding for ligands of the notch receptor, are expressed in nascent neurons and probably also in nascent hair cells (Dornseifer et al. 1997; Haddon et al. 1998). According to the lateral inhibition hypothesis (Corwin et al. 1991; Lewis 1991), the nascent hair cells, by expressing the delta protein, would inhibit their neighbors from becoming hair cells, "forcing" them to be supporting cells instead (Haddon et al. 1998). Lunatic fringe (encoded by Lnfg) is another component of the notch pathway, which seems to play a role in the commitment of the inner hair cell precursors. Unlike delta, Lfng is expressed in nonsensory supporting cells of the mouse cochlea (Zhang et al. 2000). Retinoic acid and retinoid receptors also seem to play a role in early developmental events including hair cell proliferation and commitment (Represa et al. 1990; Raz and Kelley 1997; Pasqualetti et al. 2001). Previously known zebrafish genes playing a role in inner ear innervation were also found among the sequences of our database, including BETA2/NeuroD1, which encodes NeuroD1, a transcription factor expressed in both sensory ganglia and sensory epithelia of the developing inner ear. It is implicated in neuroblast delamination from the otic vesicle epithelium. BETA2/NeuroD1 was the first gene shown to regulate neuronal and sensory cell differentiation in both the auditory and vestibular systems (Liu et al. 2000). The aforementioned genes validate the present database as a valuable tool to decipher the molecular basis of inner ear development. The remaining 548 D. rerio UniGene clusters represented in this database were made of ESTs not related to any known gene or predicted protein. Putative human orthologs were identified for 919 of the $1428 D$. rerio genes or ESTs. The positions of 820 (89\%) of these human orthologs on the GoldenPath draft of the human genome were determined; 177 genes or ESTs colocalized with published loci of nonsyn- 
dromic deafness or Usher syndrome (i.e., a syndrome including sensory hearing loss) and are therefore candidate genes for these defects.

Eleven percent $(302 / 2627)$ of the sequences that showed no similarity to sequences in the $D$. rerio section of UniGene could be tentatively assigned to 302 proteins in $\mathrm{nr}$ databank, a public databank containing an exhaustive nonredundant collection of protein sequences from different species (ftp:// ftp.ncbi.nlm.nih.gov/blast/db/nr). Considering a sliding window of 100 amino acids, the numbers of sequences in each range of percentage of similarity (positivity) to proteins in $\mathrm{nr}$ were as follows: 34 sequences between $50 \%$ and $60 \%, 47$ between $61 \%$ and $70 \%, 47$ sequences between $71 \%$ and $80 \%, 71$ between $81 \%$ and $90 \%$, and 103 sequences $>90 \%$.

The remaining 2325 contigs and singletons showed no similarity with sequences in public databanks. Some of these sequences may be internal fragments of genes or ESTs already represented in public databases by their $5^{\prime}$ and $3^{\prime}$ ends. However, novel genes involved in inner ear development, including hair cell differentiation and innervation, are likely to be represented in this group.

The complete results of this study are presented in a relational database on the Web server of the Génoscope (Centre National de Séquençage). A user-friendly Web interface (http://www.genoscope.cns.fr/zie) allows easy query of the data resumed herein.

\section{METHODS}

\section{Embryo Microdissection}

Both otocysts were excised from 1000 zebrafish embryos at 20 to 30 hpf. The microdissection technique consisted of two slanting cuts as close as possible to the anterior and posterior otocyst edges, allowing the extrusion of the entire otocyst. Some surrounding tissue was inevitably carried with the otocyst.

\section{Subtracted cDNA Libraries}

Poly $(\mathrm{A})^{+}$RNA was extracted from the microdissected otocysts and from the liver of adult zebrafish. Ear and liver cDNA were produced with the CLONTECH SMART PCR cDNA synthesis kit (CLONTECH). Liver cDNA was subtracted from the ear cDNA using the CLONTECH PCR-Select cDNA subtraction kit. Manufacturer's instructions were followed, except that two rounds of subtraction (instead of one) were performed against liver cDNA. The efficiency of the subtraction was estimated by hybridizing samples of the cDNA pools obtained before and after subtraction with a ${ }^{32}$ P-labeled zebrafish-actin probe. Signals analyzed visually showed $\sim 90 \%$ removal of housekeeping cDNAs. After subtraction, cDNA was amplified and the resulting PCR products were run on agarose gel. Three batches, containing fragments with sizes ranging from 300 to $600 \mathrm{bp}$, 600 to $1 \mathrm{Kbp}$, and >1 kbp, respectively, were eluted and subcloned in the pMOS vector (Amersham Pharmacia Biotech Europe $\mathrm{GmbH}$ ). Ten thousand independent clones were grown and frozen, and plasmid DNA was extracted for sequencing.

\section{High-Throughput Sequencing}

The reactions were performed with a Big Dye terminator cycle sequencing kit and analyzed by an ABI-377 XL automated sequencer (Applied Biosystems). Sequences were base-called using the program PHRED.

\section{Assembling Sequences}

The sequences of the cloning vectors and adaptors were removed from the selected high-quality parts of 21,848 reads in the cDNA library from the $D$. rerio inner ear region. Sequences $<100$ bases were discarded. The resulting 17,468 trimmed sequences were masked for repeated sequences using RepeatMasker (A.F. Smit and P. Green, unpubl. data. http:// repeatmasker. genome.washington.edu/cgi-bin/RM2_req.pl) and the $D$. rerio repeats library. Unmasked versions of these 17,468 nonassembled sequences were submitted to EMBL (accession nos. AL714032 to AL731531). Mate pairs (i.e., paired clone ends) were fused when a significant overlap was detected, generating what we call meta-sequences. For sequence assembling, meta-sequences or remaining single reads sharing at least $95 \%$ identity over 100 bases (as estimated with BLASTN) were clustered with the Single Linkage clustering algorithm, implemented in the software LASSAP (Gene-it), and each cluster was analyzed with PHRAP version 11.0 for constructing contig sequences. The final database contained 4694 contigs and singletons averaging 530 bases.

\section{Sequence Comparison Against Public Databanks}

The 4694 contigs and singletons were used as queries for BLASTN (default parameters) searches against the section $D$. rerio of UniGene (ftp://ftp.ncbi.nlm.nih.gov/repository/ UniGene/Dr.seq.all). Sequences were considered identical if they shared at least $95 \%$ identity in a window of at least 100 bases.

The translations in the six phases of the 2627 sequences in this database without significant similarity with sequences in $D$. rerio UniGene clusters were compared to $\mathrm{nr}$ (ftp:// ftp.ncbi.nlm.nih.gov/blast/db/nr). In this case, any match with at least $50 \%$ positivity in a window of at least 100 amino acids was considered significant.

\section{Further Sequence Annotation}

A Perl script was written that retrieves the most probable putative human ortholog for each $D$. rerio gene or EST present in our database from the HomoloGene table (ftp://ftp.ncbi.nlm. nih.gov/pub/HomoloGene/) and then parses UniGene annotations for zebrafish and human clusters (gene name, title, linkage group/chromosome, tissue expression, and reference sequence). In the next step, the script retrieves the location of these human orthologs on the GoldenPath (UCSC Human Genome Project Working Draft, December 22, 2001, assembly hg9; tables refGene.txt and all_est.txt available at http:// genome.cse.ucsc.edu/) and compares them to the nucleotide positions of the published loci for nonsyndromic deafness and Usher syndrome (nucleotide positions of delimiting markers being previously extracted from table stsMap.txt of GoldenPath). Co-located genes are labeled. Finally, the script screens our database for genes known to be expressed in the inner ear during the developmental stage this study focused on Holme et al.'s "table of gene expression in the developing ear" (http://www.ihr.mrc.ac.uk/hereditary/genetable/ index.shtml/).

The results of sequence comparison and further annotation were organized in a relational database using MYSQL. A user-friendly Web-based interface was programmed, allowing easy query of all the data of this study (http://www. genoscope.cns.fr/zie)

\section{ACKNOWLEDGMENTS}

We thank Sylvie Nouaille for technical help in microdissection of fish embryos; Eric Pelletier, Olivier Jaillon, and Abel Ureta Vidal for assistance in sequence analysis; and Jacqueline Levilliers for critical reading of this manuscript. This study was supported by the European Commission grant QLG2-CT1999-00988, and the Fondation pour la Recherche Médicale grant ARS 2000.

The publication costs of this article were defrayed in part by payment of page charges. This article must therefore be 
hereby marked "advertisement" in accordance with 18 USC section 1734 solely to indicate this fact.

\section{REFERENCES}

Abdelhak, S., Kalatzis, V., Heilig, R., Compain, S., Samson, D., Vincent, C., Weil, D., Cruaud, C., Sahly, I., Leibovici, M., et al. 1997. Human homologue of the Drosophila eyes absent gene underlies branchio-oto-renal (BOR) syndrome and identifies a novel gene family. Nat. Genet. 15: 157-164.

Amsterdam, A., Burges, S., Chen, W., Farrington, S., Golling, G., Haldi, M., Hopkins, N., Sun, Z., and Townsend, K. 1999. A large-scale insertional mutagenesis screen in zebrafish. Genes \& Dev. 13: 2713-2724.

Cohen-Salmon, M., El-Amraoui, A., Leibovici, M., and Petit, C. 1997. Otogelin: A glycoprotein specific to the acellular membranes of the inner ear. Proc. Natl. Acad. Sci. 94: 14450-14455.

Corwin, J.T., Jones, J.E., Katayama, A., Kelley, M.W., and Warchol, M.E. 1991. Hair cell regeneration: The identities of progenitor cells, potential triggers and instructive cues. Ciba Found. Symp. 160: $103-130$.

Deol, M. 1968. Inherited diseases of the inner ear in man in the light of studies on the mouse. I. Med. Genet. 5: 137-154. . 1970. The relationship between abnormalities of pigmentation and of the inner ear. Proc. R. Soc. Lond. Biol. 175: 201-217.

- 1980. Genetic malformations of the inner ear in the mouse and man. In Morphogenesis and malformation of the ear (ed. R. Gorlin), pp. 243-261. Alan R. Liss, Inc., New York.

Dornseifer, P., Takke, C., and Campos-Ortega, J.A. 1997. Overexpression of a zebrafish homologue of the Drosophila neurogenic gene Delta perturbs differentiation of primary neurons and somite development. Mech. Dev. 63: 159-171.

Ekker, M., Akimenko, M.A., Bremiller, R., and Westerfield, M. 1992. Regional expression of three homeobox transcripts in the inner ear of zebrafish embryos. Neuron 9: 27-35.

Ernest, S., Rauch, G.-J., Haffter, P., Geisler, R., Petit, C., and Nicolson, T. 2000. Mariner is defective in myosin VIIA: A zebrafish model for human hereditary deafness. Hum. Mol. Genet. 9: 2189-2196.

Gates, M.A., Kim, L., Egan, E.S., Cardozo, T., Sirotkin, H.I., Dougan, S.T., Laskari, D., Abagyan, R., Schier, A.F., and Talbot, W.S. 1999 A genetic linkage map for zebrafish: Comparative analysis of genes and expressed sequences. Genome Res. 9: 334-347.

Geisler, R., Rauch, G.-J., Baier, H., van Bebber, F., Bross, L., Dekens, M.P.S., Finger, K., Fricke, C., Gates, M.A., and Geiger, H. 1999. A radiation hybrid map of the zebrafish genome. Nat. Genet. 23: 86-89.

Gibson, F., Walsh, J., Mburu, P., Varela, A., Brown, K.A., Antonio, M., Beisel, K.W., Steel, K.P., and Brown, S.D.M. 1995. A type VII myosin encoded by the mouse deafness gene Shaker-1. Nature 374: $62-64$.

Haddon, C. and Lewis, J. 1996. Early ear development in the embryo of the zebrafish, Danio rerio. J. Comp. Neurol. 365: 113-128.

Haddon, C., Jiang, Y.-J., Smithers, L., and Lewis, J. 1998. Delta-Notch signalling and the patterning of sensory cell differentiation in the zebrafish ear: Evidence from the mind bomb mutant. Development 125: 4637-4644.

Haffter, P., Granato, M., Brand, M., Mullins, M.C., Hammerschmidt, M., Kane, D.A., Odenthal, J., van Eeden, F.J.M., Jiang, Y.-J., Heisenberg, C.-P., et al. 1996. The identification of genes with unique and essential functions in the development of the zebrafish, Danio rerio. Development 123: 1-36.

Heller, S., Sheane, C.A., Javed, Z., and Hudspeth, A.J. 1998. Molecular markers for cell types of the inner ear and candidate genes for hearing disorders. Proc. Natl. Acad. Sci. 95: $11400-11405$.

Holme, R.H., Bussoli, T.J., and Steel, K.P. 2002. Table of gene expression in the developing ear. http://www.ihr.mrc.ac.uk/hereditary/genetable/index.shtml

Hukriede, N.A., Joly, L., Tsang, M., Miles, J., Tellis, P., Epstein, J.A., Barbazuk, W.B., Li, F.N., Paw, B., Postlethwait, J.H. et al. 1999. Radiation hybrid mapping of the zebrafish genome. Proc. Natl. Acad. Sci. 96: 9745-9750.

Kalatzis, V., Sahly, I., El-Amraoui, A., and Petit, C. 1998. Eya1 expression in the developing ear and kidney: Towards the understanding of the pathogenesis of branchio-oto-renal (BOR) syndrome. Dev. Dyn. 213: 486-499.

Knapik, E.W., Goodman, A., Ekker, M., Chevrette, M., Delgado, J.,
Neuhauss, S., Shimoda, N., Driever, W., Fishman, M.C., and Jacob, H.J. 1998. A microsatellite genetic linkage map for zebrafish (Danio rerio). Nat. Genet. 18: 338-343.

Lewis, J. 1991. Rules for the production of sensory cells. Regeneration of vertebrate sensory cell. Ciba Found. Symp. 160: $25-39$.

Liu, M., Pereira, F.A., Price, S.D., Chu, M.J., Shope, C., Himes, D., Eatock, R.A., Brownell, W.E., Lysakowski, A., and Tsai, M.J. 2000. Essential role of BETA2/NeuroD1 in development of the vestibular and auditory systems. Genes \& Dev. 14: 2839-2854.

Malicki, J., Schier, A.F., Solnica-Krezel, L., Stemple, D.L., Neuhauss, S.C.F., Stainier, D.Y.R., Abdelilah, S., Rangini, Z., Zwartkruis, F., and Driever, W. 1996. Mutations affecting development of the zebrafish ear. Development 123: 275-283.

Pasqualetti, M., Neun, R., Davenne, M., and Rijli, F.M. 2001. Retinoic acid rescues inner ear defects in Hoxa1 deficient mice. Nat. Genet. 29: 34-39.

Platt, C. 1993. Zebrafish inner ear sensory surfaces are similar to those in goldfish. Hearing Res. 65: 133-140.

Popper, A.N. and Fay, R.R. 1993. Sound detection and processing by fish: Critical review and major research questions. Brain Behav. Ecol. 41: 14-38.

Postlethwait, J.H., Yan, Y.-L., Gates, M.A., Horne, S., Amores, A., Brownlie, A., Donovan, A., Egan, E.S., Force, A., Gong, Z. et al. 1998. Vertebrate genome evolution and the zebrafish gene map. Nat. Genet. 18: 345-349.

Raz, Y. and Kelley, M.W. 1997. Effects of retinoid and thyroid receptors during development of the inner ear. Semin. Cell Dev. Biol. 8: $257-264$.

Represa, J., Sanchez, A., Miner, C., Lewis, J., and Giráldez, F. 1990. Retinoic acid modulation of the early development of the inner ear is associated with control of $c$-fos expression. Development 110: $1081-1090$.

Rinkwitz-Brandt, S., Arnold, H.H., and Bober, E. 1996. Regionalized expression of Nkx5-1, Nkx5-2, Pax2 and sek genes during mouse inner ear development. Hearing Res. 99: 129-138.

Robertson, N.G., Khetarpal, U., Gutierrez-Espeleta, G.A., Bieber, F.R., and Morton, C.C. 1994. Isolation of novel and known genes from a human fetal cochlear cDNA library using subtractive hybridization and differential screening. Genomics 23: $42-50$.

Robertson, N.G., Lu, L., Heller, S., Merchant, S.N., Eavey, R.D., McKenna, M., Nadol Jr., J.B., Miyamoto, R.T., Linthicum, Jr. F.H., Lubianca Neto, J.F., et al. 1998; Mutations in a novel cochlear gene cause DFNA9, a human nonsyndromic deafness with vestibular dysfunction. Nat. Genet. 20: 299-303.

Robinson, G.W. and Mahon, K.A. 1994. Differential and overlapping expression domains of $D l x-2$ and $D l x-3$ suggest distinct roles for Distal-less homeobox genes in craniofacial development. Mech. Dev. 48: 199-215.

Sahly, I., Andermann, P., and Petit, C. 1999. The zebrafish eya1 gene and its expression pattern during embryogenesis. Dev. Genes Evol. 209: 399-410.

Shimoda, N., Knapik, E.W., Ziniti, J., Sim, C., Yamada, E., Kaplan, S., Jackson, D., de Sauvage, F., Jacob, H., and Fishman, M.C. 1999. Zebrafish genetic map with 2000 microsatellite markers. Genomics 58: 219-232.

Simmler, M.-C., Cohen-Salmon, M., El-Amraoui, A., Guillaud, L., Benichou, J.-C., Petit, C., and Panthier, J.-J. 2000. Targeted disruption of Otog results in deafness and severe imbalance. Nat. Genet. 24: $139-143$.

Steel, K.P. 1995. Inherited hearing defects in mice. Annu. Rev. Genet. 29: $675-701$.

Streisinger, G. 1984. Attainment of minimal biological variability and measurements of genotoxicity: Production of homozygous diploid zebra fish. Natl. Cancer Inst. Monogr. 65: 53-58.

Streisinger, G., Singer, F., Walker, C., Knauber, D., and Dower N. 1986. Segregation analyses and gene-centromere distances in zebrafish. Genetics 112: 311-319.

Streisinger, G., Walker, C., Dower, N., Knauber, D., and Singer, F. 1981. Production of clones of homozygous diploid zebra fish (Brachydanio rerio). Nature 291: 293-296.

Torres, M. and Giráldez, F. 1998. The development of the vertebrate inner ear. Mech. Dev. 71: 5-21.

Verpy, E., Leibovici, M., Zwaenepoel, I., Liu, X.-Z., Gal, A., Salem, N., Mansour, A., Blanchard, S., Kobayashi, I., Keats, B.J.B., et al. 2000. A defect in harmonin, a PDZ domain-containing protein expressed in the inner ear sensory hair cells, underlies Usher syndrome type 1C. Nat. Genet. 26: 51-55.

Verpy, E., Masmoudi, S., Zwaenepoel, I., Leibovici, M., Hutchin, T.P.,

\section{Genome Research}


Del Castillo, I., Nouaille, S., Blanchard, S., Laine, S., Popot, J.L., et al. 2001. Mutations in a new gene encoding a protein of the hair bundle cause non-syndromic deafness at the DFNB16 locus. Nat. Genet. 29: 345-349.

Waterman, R.E. and Bell, D.H. 1984. Epithelial fusion during early semicircular canal formation in the embryonic zebrafish, Brachydanio rerio. Anat. Rec. 210: 101-114.

Weil, D., Blanchard, S., Kaplan, J., Guilford, P., Gibson, F., Walsh, J. Mburu, P., Varela, A., Levilliers, J., Weston, M.D., et al. 1995. Defective myosin VIIA gene responsible for Usher syndrome type 1B. Nature 374: 60-61.

Whitfield, T.T., Granato, M., van Eeden, F.J.M., Schach, U., Brand, M., Furutani-Seiki, M., Haffter, P., Hammerschmidt, M. Heisenberg, C.-P., Jiang, Y.-J., et al. 1996. Mutations affecting development of the zebrafish inner ear and lateral line. Development 123: 241-254.

Yasunaga, S., Grati, M., Cohen-Salmon, M., El-Amraoui, A., Mustapha, M., Salem, N., El-Zir, E., Loiselet, J., and Petit, C. 1999. A mutation in OTOF, encoding otoferlin, a FER-1 like protein, causes DFNB9, a nonsyndromic form of deafness. Nat. Genet. 21: 363-369.
Zhang, N., Martin, G.V., Kelley, M.W., and Gridley, T. 2000. A mutation in the Lunatic fringe gene suppresses the effects of a Jagged 2 mutation on inner hair cell development in the cochlea. Curr. Biol. 10: 659-662.

\section{WEB SITE REFERENCES}

ftp://ftp.ncbi.nlm.nih.gov/repository/UniGene/Dr.seq.all; National Center for Biotechnology Information (NCBI)

$\mathrm{ftp}: / /$ ftp.ncbi.nlm.nih.gov/blast/db/nr; Non-redundant (NCBI)

$\mathrm{ftp}$ //ftp.ncbi.nlm.nih.gov/pub/HomoloGene; HomoloGene (NCBI).

http://genome.cse.ucsc.edu; UCSC Human Genome Project Working Draft, December 22, 2001, assembly hg9.

http://repeatmasker.genome.washington.edu/cgi-bin/RM2_req.pl; RepeatMasker software.

http://www.ihr.mrc.ac.uk/hereditary/genetable/index.shtml; Table of gene expression in the developing ear.

Received December 13, 2001; accepted in revised form April 5, 2002. 


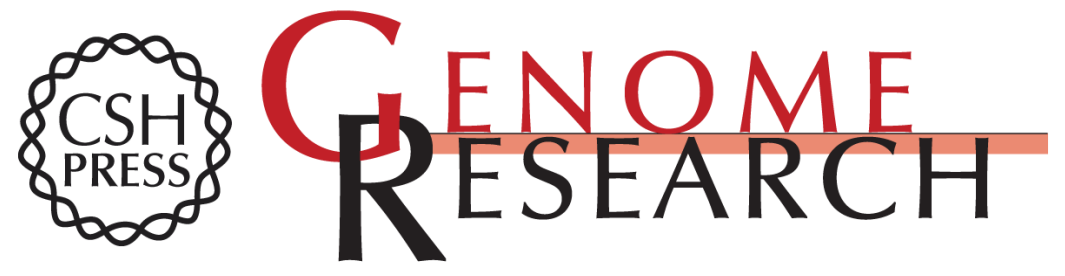

\section{A Subtracted cDNA Library from the Zebrafish (Danio rerio) Embryonic Inner Ear}

Roney S. Coimbra, Dominique Weil, Phillipe Brottier, et al.

Genome Res. 2002 12: 1007-1011

Access the most recent version at doi:10.1101/gr.227502

References This article cites 45 articles, 13 of which can be accessed free at:

http://genome.cshlp.org/content/12/6/1007.full.html\#ref-list-1

\section{License}

Email Alerting Receive free email alerts when new articles cite this article - sign up in the box at the Service top right corner of the article or click here.

\section{Affordable, Accurate Sequencing.}

